\title{
KSHV co-infection down-regulates HPV16 E6 and E7 from cervical cancer cells
}

\author{
Lu Dai ${ }^{1,2,4}$, Yueyu Cao², Wei Jiang ${ }^{3}$, Jovanny Zabaleta ${ }^{5}$, Zhongmin Liư², Jing Qiao ${ }^{1}$, \\ Zhiqiang Qin ${ }^{1,2,4}$ \\ ${ }^{1}$ Department of Pediatrics, East Hospital, Tongji University School of Medicine, Shanghai 200120, China \\ ${ }^{2}$ Research Center for Translational Medicine and Key Laboratory of Arrhythmias, East Hospital, Tongji University School of \\ Medicine, Shanghai 200120, China \\ ${ }^{3}$ Department of Microbiology and Immunology, Division of Infectious Diseases, Department of Medicine, Medical University \\ of South Carolina, Charleston, SC 29425, USA \\ ${ }^{4}$ Departments of Genetics Louisiana State University Health Sciences Center, Louisiana Cancer Research Center, New \\ Orleans, LA 70112, USA \\ ${ }^{5}$ Pediatrics, Louisiana State University Health Sciences Center, Louisiana Cancer Research Center, New Orleans, LA 70112, \\ USA
}

Correspondence to: Jing Qiao, email: qiaoj123@hotmail.com

Zhiqiang Qin, email: zqin@Isuhsc.edu

Keywords: $H P V, K S H V, E 6, E 7$, microRNA

Received: February 14, $2017 \quad$ Accepted: March 07, $2017 \quad$ Published: March 15, 2017

Copyright: Dai et al. This is an open-access article distributed under the terms of the Creative Commons Attribution License (CC-BY), which permits unrestricted use, distribution, and reproduction in any medium, provided the original author and source are credited.

\section{ABSTRACT}

High-risk human papillomavirus (HPV) infection is the etiological agent of some malignancies such as cervical, oral and oropharyngeal cancers. Kaposi sarcomaassociated herpesvirus (KSHV) represents a principal causative agent of several human cancers arising in those immunocompromised patients. Interestingly, KSHV DNA has been detected in the oral cavity and the female genital tract, although its detection rate in cervical samples is very low and few reports are about KSHV/HPV co-infection. Therefore, it remains unclear about the role of KSHV co-infection in the development of HPV-related neoplasias. In the current study, we report that HPV16integrated cervical cancer cell-line $\mathrm{SiHa}$ is susceptible to KSHV latent infection and replication. We also have found that KSHV infection or viral latent proteins are capable of reducing HPV16 E6/E7 expression through the manipulation of cellular microRNA function. Array analysis indicates that KSHV infection induces some inflammatory cytokines/chemokines production as well as up-regulates a series of interferoninduced genes expression, which may facilitate host immune defense system attacking these co-infected cells and clearance of viruses. Together, our data have provided possible explanations for very low detection rate of KSHV shedding as well as of KSHV/HPV co-infection in cervical samples and/or cervical cancer cells.

\section{INTRODUCTION}

Cervical cancer represents one of the most common malignancies in females worldwide. The pathogenesis of cervical cancer occurs following persistent infection with high-risk human papillomavirus (HPV) such as subtype 16 and 18 [1]. High-risk HPV-encoded E6 and E7 proteins are major viral oncoproteins which are closely associated with human cervical carcinogenesis [2]. E6 and E7 can bind to the p53 and retinoblastoma $(\mathrm{Rb})$ family proteins, respectively, resulting in the regulation of cell cycle and final transformation [3]. In addition, high-risk HPV infection is also prevalent in oral cavity and related to oral and oropharyngeal cancer development [4-6].

Another oncogenic virus, Kaposi sarcomaassociated herpesvirus (KSHV) represents a principal causative agent of several human cancers arising in those immunocompromised patients, including Kaposi's Sarcoma (KS), Primary effusion lymphoma (PEL) and Multicentric Castleman's disease (MCD) [7-9]. Published literatures have reported that KSHV DNA sequences can be detected in the prostate [10], semen [11], oral cavity 
[12] and the female genital tract $[13,14]$. Moreover, person-to-person transmission of KSHV is thought to occur primarily through exchange of oropharyngeal secretions [15]. In contrast to the high prevalence of KSHV shedding in oral cavity, the detection rate of KSHV DNA or virus infection in cervical samples are relatively low $(<2 \%)$, even in those high-risk population such as sex workers and HIV+ persons [14, 16]. Furthermore, currently there are few studies reporting the co-infection of KSHV and HPV in cervical samples and/or cervical cancer cells. Therefore, it remains unclear about the role of KSHV co-infection in the development of HPV-related neoplasias. In the current study, we tested the susceptibility of HPV16-integrated cervical cancer cell-line $\mathrm{SiHa}$ to KSHV infection, replication and their impacts on HPVencoded oncoproteins expression. Interestingly, we found that KSHV de novo infection or viral latent proteins significantly reduced HPV16 E6/E7 expression through the manipulation of cellular microRNA function. We also found that KSHV infection induced some inflammatory cytokines/chemokines production as well as up-regulated a series of interferon-induced genes expression, which may facilitate host immune defense system attacking these co-infected cells and clearance of viruses.

\section{RESULTS}

\section{KSHV can establish latent infection within $\mathrm{SiHa}$ cells and possess replicative potential}

Latent KSHV infection is dependent upon intranuclear expression of KSHV-encoded latency associated nuclear antigen (LANA), which tethers viral episome to host cell chromatin [17]. To first determine whether SiHa cells are susceptible to KSHV infection, we incubated them with purified KSHV virions and used immunofluorescence (IFA) to quantify LANA expression within individual cells. The confocal microscopy images revealed LANA expression within $>95 \%$ of $\mathrm{SiHa}$ cell nuclear following $72 \mathrm{~h}$ post infection (p.i.) with an MOI $\sim 10$, while no LANA dots observed in the control mock cells (Figure 1). To further validate replicative potential of these viruses in latently infected SiHa cells, we treated infected cells with valproic acid (VA) for 5 days, a common chemical inducing viral lytic reactivation [18]. Then we used qRT-PCR to quantify the transcripts of representative viral latent and lytic genes. Our results indicated VA treatment greatly induced different viral lytic genes expression (RTA, vGPCR, K8.1 and ORF57), while dramatically reducing latent gene LANA expression from infected SiHa cells (Figure 2A). Immunoblots analysis confirmed the elevated expression of $\mathrm{K} 8.1$, one of lytic proteins by VA (Figure 2B). Furthermore, we found that VA induced infected $\mathrm{SiHa}$ cells release of infectious KSHV particles in culture supernatants, as demonstrated by increased LANA expression within fresh KSHV- naïve cells following their exposure to VA-treated $\mathrm{SiHa}$ supernatants (Figure 2C). Together, our data demonstrate that $\mathrm{SiHa}$ are susceptible to KSHV latent infection and these viruses are capable of self-replication once stimulated to lytic reactivation.

\section{Global signature of cellular cytokine/chemokine altered within $\mathrm{KSHV}$-infected SiHa cells}

By using a cytokine/chemokine array, we identified a global signature altered within KSHV-infected $\mathrm{SiHa}$ when compared to the control mock cells. We found that KSHV infection increased several inflammatory factors production from $\mathrm{SiHa}$ cells, including Chemokine (C-X-C motif) ligand 1 (CXCL1), Interleukin 6 (IL-6), Plasminogen activator inhibitor-1 (PAI-1), Chemokine (C-C motif) ligand 5 (CCL5), Interleukin 8 (IL-8) and Macrophage migration inhibitory factor (MIF) (Figure 3). Our additional data have demonstrated KSHV infection does not affect $\mathrm{SiHa}$ cell growth and viability (Supplementary Figure 1), therefore which is not responsible for the increased cytokines/chemokines production we have observed.

\section{The down-regulation of HPV16 E6 and E7 by KSHV and/or viral latent proteins}

SiHa cells contain an integrated HPV16 genome, and E6/E7 represent major HPV-encoded oncogenic proteins $[1,2]$. Interestingly, our data indicated that KSHV infection significantly reduced both E6 and E7 expression from SiHa cells (Figure 4A). In contrast to this, VA treatment abolished this reduction, implying the important role of $\mathrm{KSHV}$-encoded latent proteins in this regulation (Figure 4B). To prove that, we ectopically expressed LANA and vFLIP (viral FLICE inhibitory protein), two major KSHV-encoded latent proteins in SiHa cells using the recombinant constructs, respectively. We found that ectopic expression of either LANA or vFLIP effectively down-regulated E6 and E7 expression in a dose-dependent manner (Figure 4C-4D).

\section{The up-regulation of miR129-5p is required for KSHV/viral proteins reducing E6 and E7 expression}

We subsequently sought to determine how KSHV infection and/or viral latent proteins reducing E6 and E7 expression. One of potential mechanisms is through cellular microRNAs such as miRNA129-5p and miRNA331-3p [19, 20]. Zhang et al. have reported that interferon- $\beta$ treatment can induce miRNA129-5p, while its levels gradually decrease with the development of cervical intraepithelial lesions and correlate with E6 and E7 expression [19]. Another group has found that miRNA331$3 p$ can suppress cervical cancer cell proliferation and E6/ 
E7 expression by targeting Neuropilin 2 (NRP2) [20]. So we tested these 2 cellular microRNAs expression in $\mathrm{SiHa}$ cells. We found that either KSHV infection or ectopic expression of LANA or vFLIP significantly increased miRNA129-5p but not miRNA331-3p from SiHa cells (Figure 5A-5B). We next used specific miRNA129-5p inhibitor to block its activities (Supplementary Figure 2), which effectively restored E6/E7 expression from KSHVinfected or LANA-/vFLIP-transfected SiHa cells (Figure $5 \mathrm{C}-5 \mathrm{E})$. These data demonstrate that miRNA129-5p but not miRNA331-3p is required for $\mathrm{KSHV}$ and/or viral latent proteins reducing E6/E7 expression from $\mathrm{SiHa}$ cells.

\section{Transcriptomic analysis of the gene profile altered in KSHV-infected SiHa cells}

We used the HumanHT-12 v4 Expression BeadChip (Illumina), which contains more than 47,000 probes derived from the NCBI RefSeq Release 38 and other sources, to study the gene profile altered within KSHV-infected SiHa cells. We found that 71 genes were significantly up-regulated and 27 were down-regulated $(\geq 2$ fold and $p<0.05)$ within KSHV-infected SiHa cells when compared to the control mock cells (Tables 1 and 2). For validation of microarray analysis, we next selected 5 candidate genes from Tables 1 and 2, respectively, to perform qRT-PCR analysis. Our results indicated that all of the 10 selected genes were significantly altered in a manner comparable to those found in the microarray data, demonstrating the credibility of our results. Specifically, IFI27, KRT34, IL6, CEMIP and MXI were significantly up-regulated, while $A K R 1 C 3, S E M A 3 C$, ATOH8, ALDH3A1 and CRIP2 were significantly downregulated within KSHV-infected SiHa cells (Supplementary Figure 3). We also performed enrichment analysis of these significantly altered candidates by using the Pathway Maps, Gene Ontology (GO) Processes and Process Networks modules from Metacore Software (Thompson Reuters). Our analysis showed that these significantly altered candidates belong to several major functional categories, including cellular response to type I interferon (IFN), defense response to virus infection/replication, cellular response to stress and inflammation (Figure 6). In addition, the top 2 scored pathway maps and protein networks were also listed in Supplementary Figures 4 and 5, respectively. Notably, we found that KSHV infection induced strong cellular response to type I IFN, especially those IFN-induced genes expression. Our qRT-PCR data confirmed that a series of 10 IFN-induced genes were significantly up-regulated from KSHV-infected SiHa cells when compared to the control mock cells (Figure 7).

\section{DISCUSSION}

To our knowledge, this is the first study reporting KSHV infection and/or viral latent proteins can downregulate HPV16 E6 and E7 expression from cervical cancer cells. We also have found the underlying mechanism is through the down-regulation of at least one

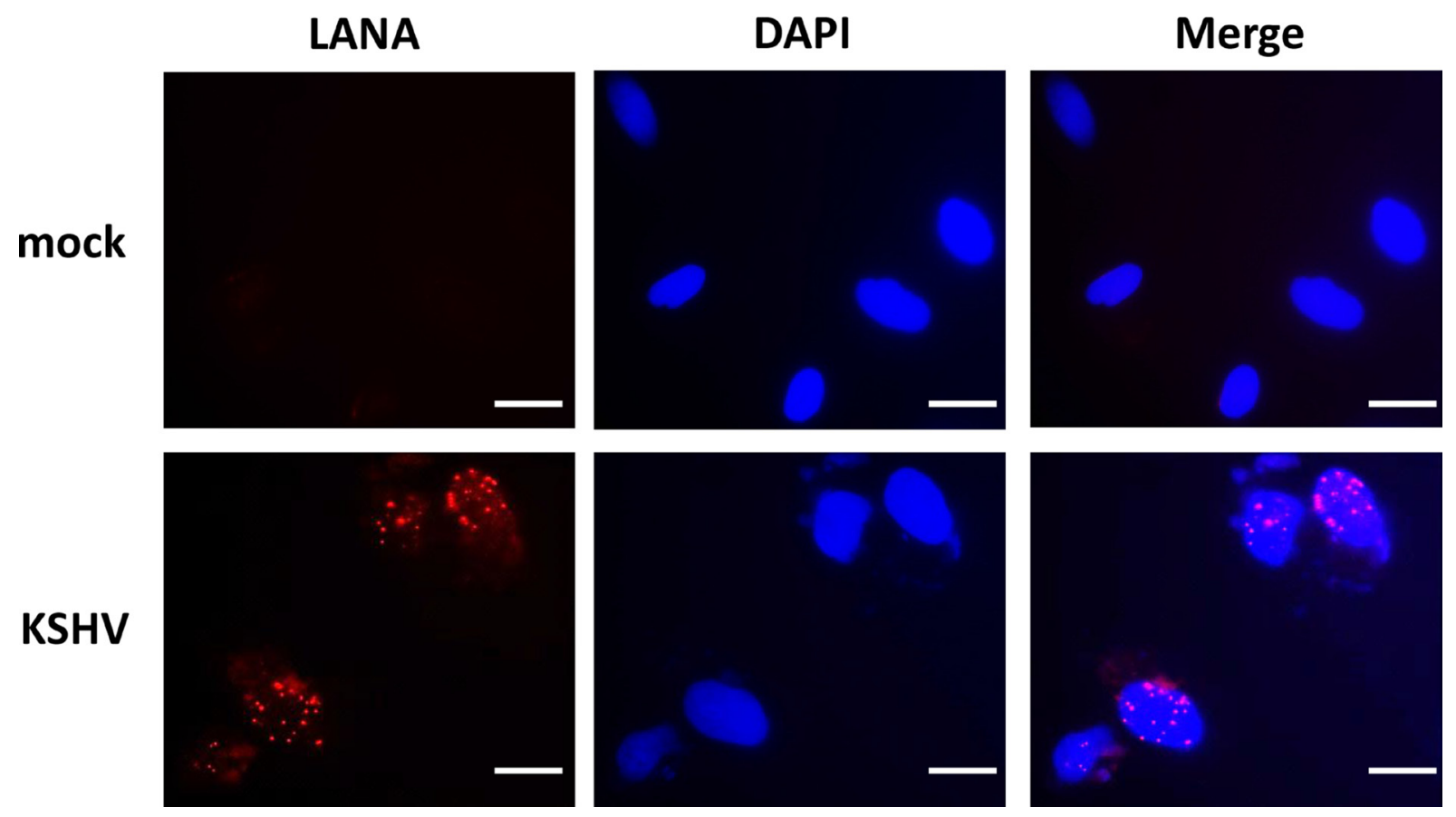

Figure 1: Establishment of latent KSHV infection within SiHa cells. SiHa were incubated with purified KSHV (MOI 10), or medium control (mock) for $2 \mathrm{~h}$. After cells were incubated for an additional $72 \mathrm{~h}$ in fresh media, immunofluorescence was performed to quantify expression of KSHV-encoded LANA as indicated by the typical intranuclear, punctate staining pattern (red dots). Nuclei were identified using DAPI (blue). Bars, $20 \mu \mathrm{m}$. 
cellular microRNA, miRNA129-5p, although some other mechanisms are possibly involved as well. Interestingly, the down-regulation of E6 and E7 expression usually causes the suppression of cervical cancer cell proliferation and the promotion of cancer cell apoptosis [19-22]. However, we do not observe that KSHV infection can affect $\mathrm{SiHa}$ cell proliferation or viability, implying that some KSHV-related factors (viral or host) may compensate
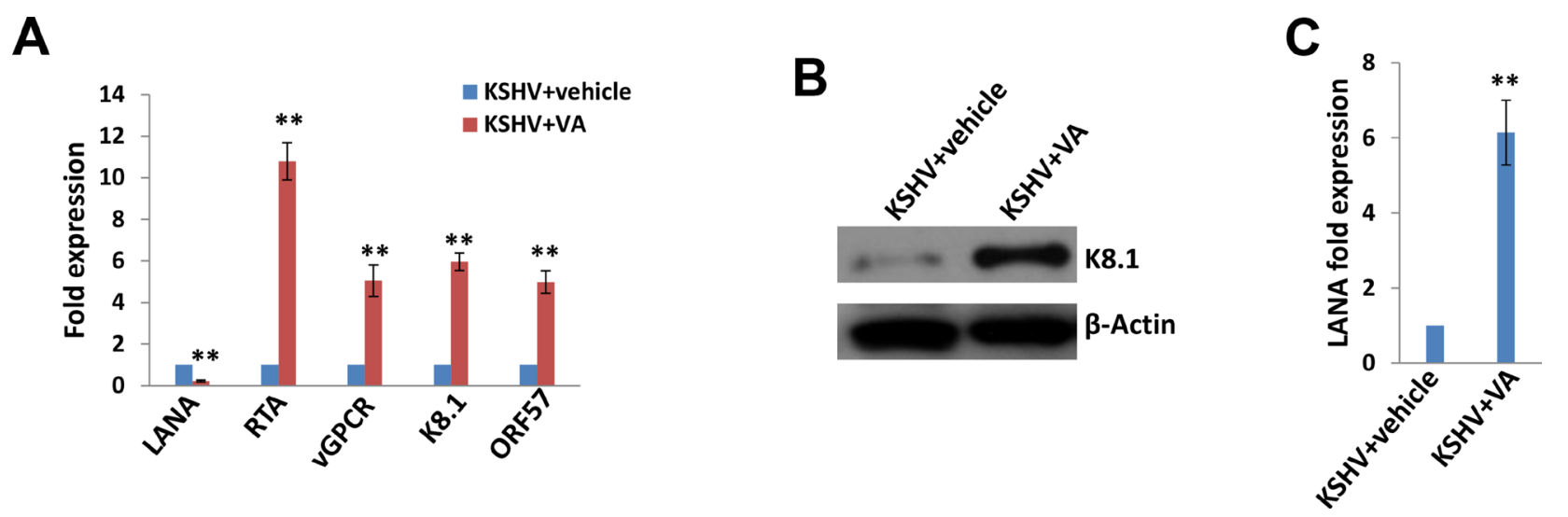

Figure 2: Induction of lytic reactivation and virus production from KSHV latently infected SiHa cells. (A-B) KSHV latently infected SiHa cells were incubated with $0.6 \mathrm{mM}$ valproic acid (VA) or vehicle for 5 days, then qRT-PCR and immunoblots were performed as described in the Methods. (C) The virion production was collected as described in the Methods, followed by infection of fresh SiHa cells. Lana transcripts were quantified by using qRT-PCR. Error bars represent the S.D. for 3 independent experiments, $* *=p<0.01$.

A
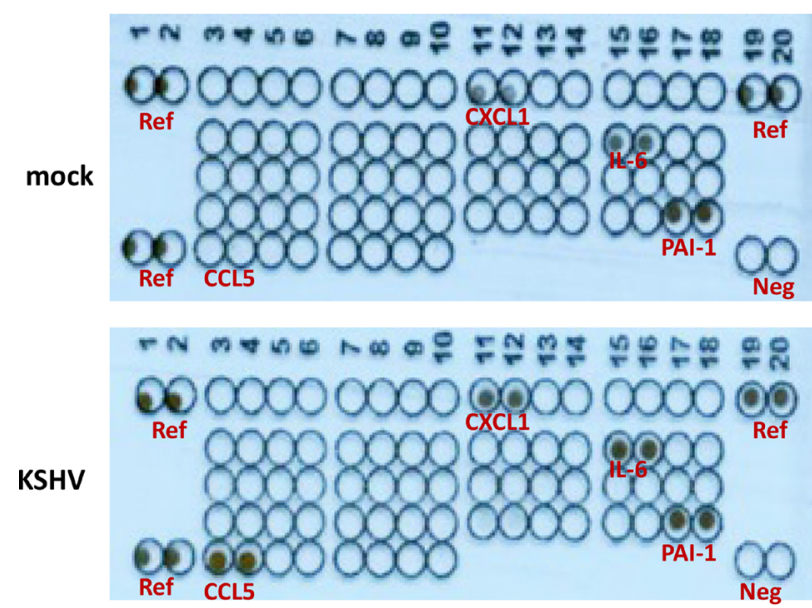

5 min
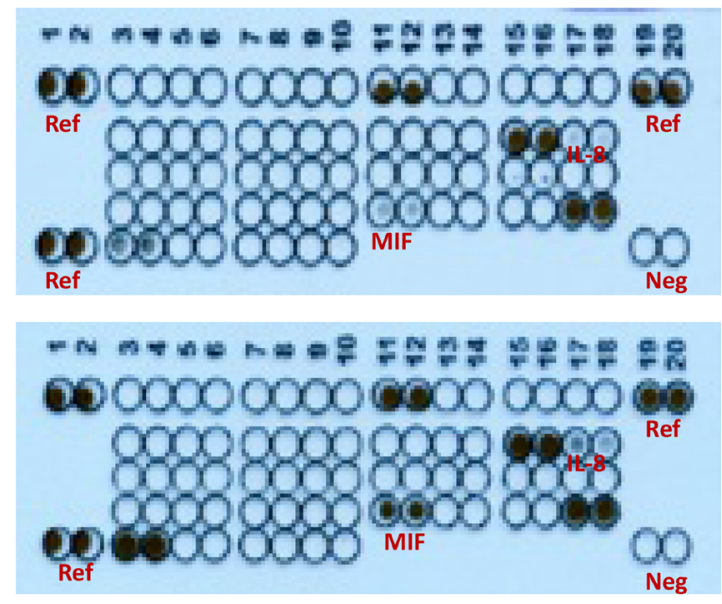

mock

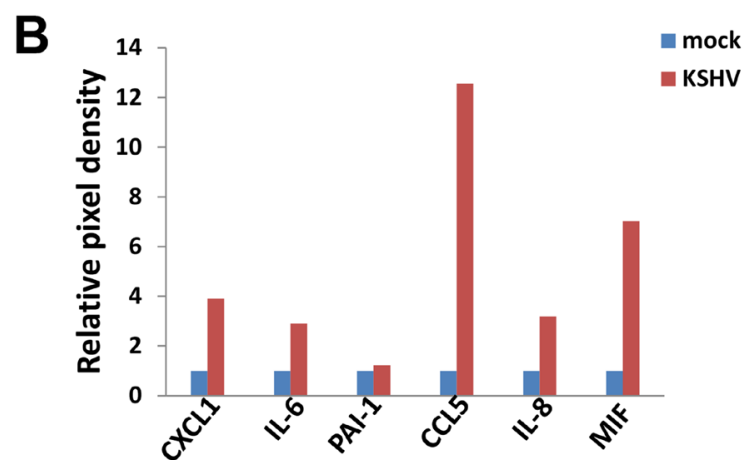

Figure 3: Cytokine/chemokine profile altered within KSHV latently infected SiHa cells. (A) SiHa were incubated with purified KSHV (MOI 10), or medium control (mock) as describe above, then the supernatants were collected and the concentrations of different cytokines/chemokines were measured as described in the Methods. (B) The density of dot-blot was scanned and quantified by using the ImageJ software. Ref: reference positive control wells; Neg: negative control wells. 
the effects of down-regulation of HPV16 E6 and E7 on cancer cell growth. Instead, we have found that KSHV infection greatly reduced $\mathrm{SiHa}$ cell invasiveness when compared to the control mock cells (data not shown). It remains unclear whether this down-regulation of E6 and E7 by KSHV is cell-line or HPV subtype specific, although we have reported that KSHV can establish latent infection within HPV18-integrated HeLa cells as well [23]. So we will test the susceptibility of other HPV+ cervical cancer cell-lines to KSHV infection, replication and their impacts on HPV-encoded oncoproteins such as E6 and E7.

One remaining question is the mechanisms through which miRNA129-5p inhibiting E6 and E7 expression. Zhang et al. have reported that the transcription factor $\mathrm{SP} 1$ is a direct downstream target of miR-129-5p in Hela cells, and SP1 expression is down-regulated significantly by over-expressed miR-129-5p [19]. Interestingly, the upstream regulatory regions of HPV-18 genes contain the SP1 binding site, which have been shown to determine E6 and E7 expression [24, 25]. So future work will try to determine whether the similar mechanisms are present in SiHa cells, or whether E6 and E7 are direct targets by miR-129-5p. Another interesting question is how KSHV infection can manipulate miR-129-5p expression in $\mathrm{SiHa}$ cells. In fact, KSHV infection or viral proteins have been found to regulate a variety of cellular microRNAs expression [26]. For instance, Tsai and colleagues have reported that KSHV-encoded K15 protein, minor form named as $\mathrm{K} 15 \mathrm{M}$, can induce cell migration and invasion through the up-regulation of cellular miR-21 and miR-31 via its conserved Src-Homology 2 (SH2)-binding motif [27]. KHSV infection and ectopic expression of vFLIP can suppress the expression of one chemokine receptor, CXCR4, through the up-regulation of cellular miR-146a expression, a miRNA that is known to bind to the 3'UTR of CXCR4 mRNA [28].

In the present study, we have found that KSHV de novo infection can induce some inflammatory cytokines/ chemokines production from $\mathrm{SiHa}$ cells. Interestingly, one of these factors, CXCL1 (also named as growth regulated oncogene 1, GRO-1), its serum levels were significantly higher in patients with cervical squamous cell carcinoma (CSCC) when compared with patients with cervical intraepithelial neoplasia (CIN) and the healthy controls [29]. However, these up-regulated inflammatory cytokines/chemokines may promote the recruitment of immune cells such as neutrophils and macrophages, enhance local inflammatory response, and finally facilitate attacking infected cells and/or the clearance of viruses. Another interesting finding in our study is that KSHV
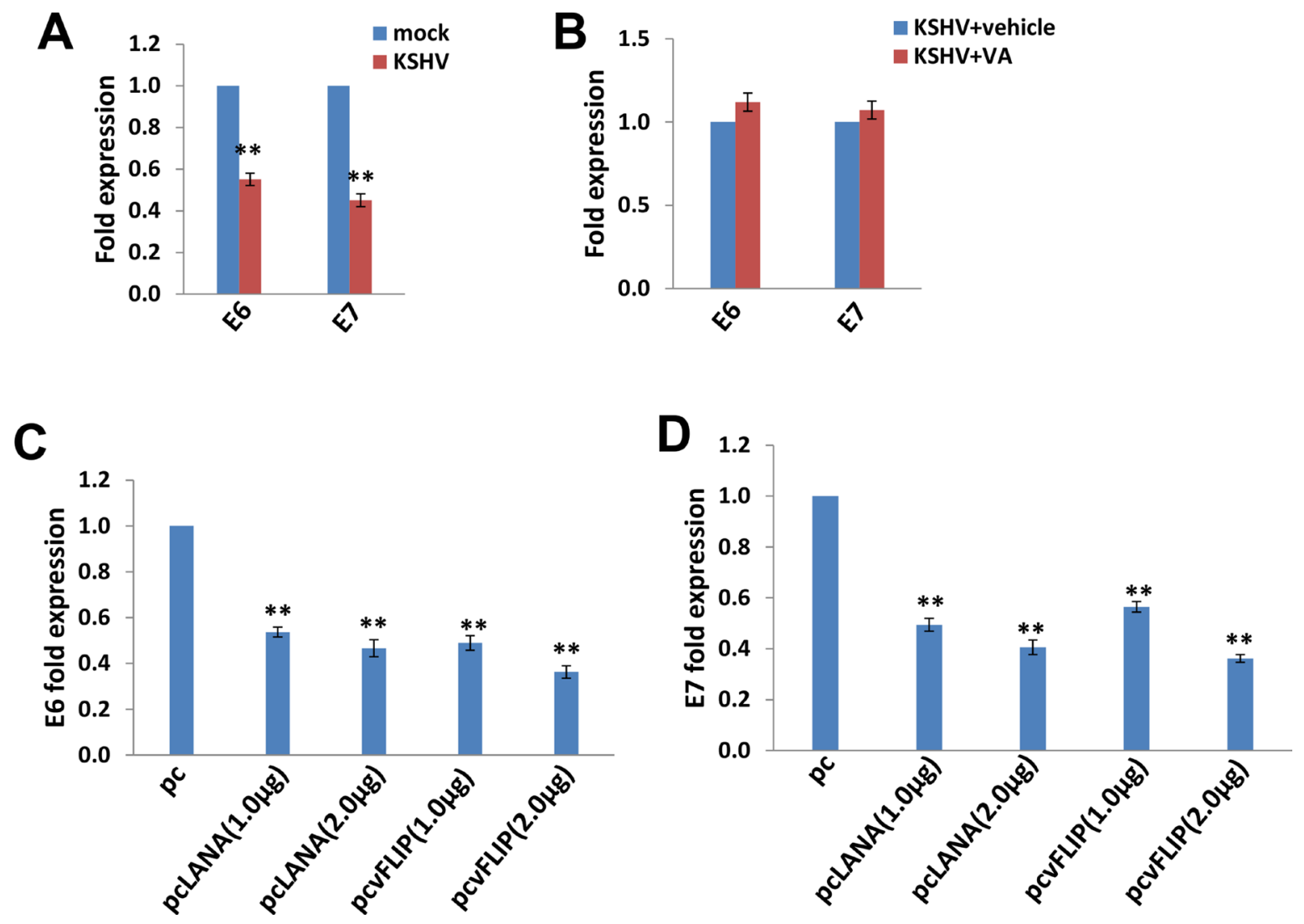

Figure 4: The down-regulation of HPV16 E6 and E7 by KSHV and/or viral latent proteins. (A-B) SiHa cells were infected by KSHV or induced lytic reactivation as described above, then gene transcripts were quantified by using qRT-PCR. (C-D) SiHa were transfected with control vector pcDNA3.1 (pc), pcDNA3.1-LANA (pcLANA) or pcDNA3.1-vFLIP (pcvFLIP) as described in the Methods, then gene transcripts were quantified by using qRT-PCR. The Error bars represent the S.D. for 3 independent experiments, $* *=p<0.01$. 
Table 1: The genes up-regulated within KSHV-infected SiHa cells

\begin{tabular}{|c|c|c|}
\hline Gene Symbol & Description & Ratio \\
\hline IFI27 & Interferon alpha-inducible protein 27 , mitochondrial & 13.24683 \\
\hline KRT34 & Keratin, type I cuticular Ha4 & 10.96868 \\
\hline IL6 & Interleukin-6 & 6.189084 \\
\hline CEMIP & Cell migration-inducing and hyaluronan-binding protein & 4.637684 \\
\hline MX2 & Interferon-induced GTP-binding protein $\mathrm{Mx} 2$ & 4.297035 \\
\hline EPSTI1 & Epithelial-stromal interaction protein 1 & 3.975506 \\
\hline RSAD2 & Radical S-adenosyl methionine domain-containing protein 2 & 3.695813 \\
\hline MX1 & Interferon-induced GTP-binding protein Mx1 & 3.616665 \\
\hline DHRS2 & Dehydrogenase/reductase SDR family member 2 , mitochondrial & 3.584949 \\
\hline CXCL8 & Interleukin-8 & 3.533108 \\
\hline HIST1H4H & Histone H4 & 3.443781 \\
\hline NT5E & 5'-nucleotidase & 3.408309 \\
\hline ISG15 & Ubiquitin-like protein ISG15 & 3.371505 \\
\hline IGF2BP2 & Insulin-like growth factor 2 mRNA-binding protein 2 & 3.326956 \\
\hline NKD2 & Protein naked cuticle homolog 2 & 3.230526 \\
\hline GADD45A & Growth arrest and DNA damage-inducible protein GADD45 alpha & 3.21191 \\
\hline OASL & 2'-5'-oligoadenylate synthase-like protein & 3.158974 \\
\hline USP18 & Ubl carboxyl-terminal hydrolase 18 & 3.0996 \\
\hline STC2 & Stanniocalcin-2 & 2.99668 \\
\hline RASD1 & Dexamethasone-induced Ras-related protein 1 & 2.964571 \\
\hline LINC00161 & long intergenic non-protein coding RNA 161 transcript & 2.940959 \\
\hline IFIT1 & Interferon-induced protein with tetratricopeptide repeats 1 & 2.915374 \\
\hline PPP1R15A & Protein phosphatase 1 regulatory subunit $15 \mathrm{~A}$ & 2.883852 \\
\hline IFIT2 & Interferon-induced protein with tetratricopeptide repeats 2 & 2.849808 \\
\hline IFI44 & Interferon-induced protein 44 & 2.807813 \\
\hline RELB & Transcription factor RelB & 2.803865 \\
\hline AP-1 & Transcription factor AP-1 & 2.746863 \\
\hline PLD6 & Mitochondrial cardiolipin hydrolase & 2.714222 \\
\hline SNHG15 & small nucleolar RNA host gene 15 transcript & 2.642434 \\
\hline PHLDA1 & Pleckstrin homology-like domain family A member 1 & 2.604317 \\
\hline IFIT3 & Interferon-induced protein with tetratricopeptide repeats 3 & 2.598199 \\
\hline RIMKLB & Beta-citrylglutamate synthase B & 2.581286 \\
\hline OAS1 & 2'-5'-oligoadenylate synthetase 1 & 2.571998 \\
\hline LRP5L & Low-density lipoprotein receptor-related protein 5-like protein & 2.488861 \\
\hline CCND1 & G1/S-specific cyclin-D1 & 2.451345 \\
\hline DUSP5 & Dual specificity protein phosphatase 5 & 2.441991 \\
\hline OLR1 & Oxidized low-density lipoprotein receptor 1 & 2.431704 \\
\hline LOC285074 & Anaphase promoting complex subunit 1 pseudogene & 2.369141 \\
\hline CCL5 & C-C motif chemokine 5 & 2.359643 \\
\hline IFI6 & Interferon alpha-inducible protein 6 & 2.327306 \\
\hline CTGF & Connective tissue growth factor & 2.324625 \\
\hline SLCO2A1 & Solute carrier organic anion transporter family member $2 \mathrm{~A} 1$ & 2.321616 \\
\hline
\end{tabular}




\begin{tabular}{llc} 
DDIT3 & DNA damage-inducible transcript 3 protein & 2.312649 \\
MAFF & Transcription factor MafF & 2.29661 \\
OTUD1 & OTU domain-containing protein 1 & 2.268293 \\
SNHG12 & Putative uncharacterized protein SNHG12 & 2.267007 \\
TUBB2B & Tubulin beta-2B chain & 2.262177 \\
ETV5 & ETS translocation variant 5 & 2.257368 \\
PMAIP1 & Phorbol-12-myristate-13-acetate-induced protein 1 & 2.244876 \\
HIST1H4D & Histone H4 & 2.243342 \\
WARS & Tryptophan--tRNA ligase, cytoplasmic & 2.23195 \\
PDP1 & [Pyruvate dehydrogenase [acetyl-transferring]]-phosphatase 1, mitochondrial & 2.217114 \\
OAS2 & 2'-5'-oligoadenylate synthetase 2 & 2.199281 \\
ASNS & Asparagine synthetase [glutamine-hydrolyzing] & 2.19681 \\
LINC01554 & Putative uncharacterized protein encoded by LINC01554 & 2.158002 \\
NR2C1 & Nuclear receptor subfamily 2 group C member 1 & 2.154326 \\
KRT16 & Keratin, type I cytoskeletal 16 & 2.144548 \\
EPB41L4A-AS1 & EPB41L4A antisense RNA 1 transcript & 2.143205 \\
GADD45B & Growth arrest and DNA damage-inducible protein GADD45 beta & 2.142809 \\
HIST1H2BC & Histone H2B type 1-C/E/F/G/I & 2.140187 \\
SLC15A3 & Solute carrier family 15 member 3 & 2.117546 \\
ERRFI1 & ERBB receptor feedback inhibitor 1 & 2.112755 \\
HIST1H4E & Histone H4 & 2.108247 \\
TAF1D & TATA box-binding protein-associated factor RNA polymerase I subunit D & 2.108209 \\
IFITM1 & Interferon-induced transmembrane protein 1 & 2.096043 \\
SNAPC4 & snRNA-activating protein complex subunit 4 & 2.059977 \\
ARNT2 & Aryl hydrocarbon receptor nuclear translocator 2 & 2.028269 \\
SNHG1 & small nucleolar RNA host gene 1 transcript & 2.01646 \\
RHOB & Rho-related GTP-binding protein RhoB & 2.015713 \\
HELZ2 & Helicase with zinc finger domain 2 & 2.015691 \\
NFIL3 & Nuclear factor interleukin-3-regulated protein & 2.014101 \\
\hline & & \\
\hline
\end{tabular}

infection up-regulates a series of IFN-induced genes expression from SiHa cells. We actually have observed similar responses within KSHV-infected human primary oral fibroblasts [30]. As we know, high-risk HPV infection down-regulates IFN $\alpha$-inducible gene expression, for example, the HPV16 E6 and E7 oncoproteins interact directly with the components of the IFN signaling pathways [31]. Since IFN has been widely used in the treatment of CIN and cervical cancer, the down-regulation of E6 and E7 expression may be responsible for the positive clinical outcomes observed with IFN treatment [19]. Therefore, our data suppose that KSHV co-infection may arouse IFN signaling pathway activities to accelerate virus clearance by host immune system. Taken together, our data have provided possible explanations for very low detection rate of KSHV shedding as well as of KSHV/ HPV co-infection in cervical samples and/or cervical cancer cells.

\section{MATERIALS AND METHODS}

\section{Cell culture and KSHV purification/infection}

Body cavity-based lymphoma cells (BCBL-1, $\left.\mathrm{KSHV}^{+} / \mathrm{EBV}^{\text {neg }}\right)$ were kindly provided by Dr. Dean Kedes (University of Virginia) and maintained in RPMI 1640 medium (Gibco) with supplements as described previously [32]. SiHa cells were purchased from ATCC and maintained in Eagle's Minimum Essential Medium (ATCC) supplemented with 10\% FBS. All cells were incubated at $37^{\circ} \mathrm{C}$ in $5 \% \mathrm{CO}_{2}$. All experiments were carried out using cells harvested at low passages $(<20)$. To obtain KSHV for infection experiments, BCBL-1 cells were incubated with $0.6 \mathrm{mM}$ valproic acid for 6 days, and purified virus was concentrated from culture supernatants and infectious titers were determined as described previously [33]. 

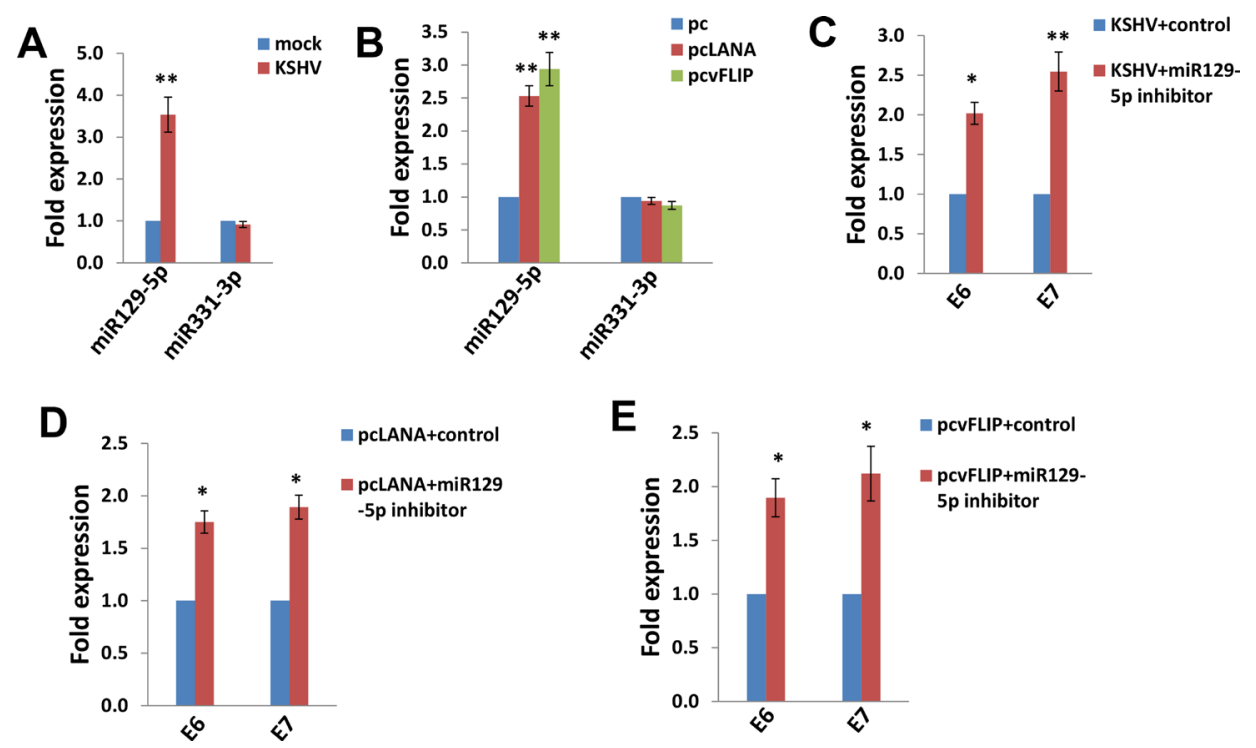

Figure 5: The up-regulation of miR129-5p is required for KSHV/viral latent proteins reducing HPV16 E6 and E7 expression. (A-B) SiHa cells were infected by KSHV or transfected using LANA or vFLIP recombinant constructs as described above, then miRNAs transcripts were quantified by using qRT-PCR. (C-E) SiHa cells were transfected with control or miR129-5p inhibitor prior to KSHV infection or ectopic expression of LANA or vFLIP, then gene transcripts were quantified by using qRT-PCR. The Error bars represent the S.D. for 3 independent experiments, $*=p<0.05, * *=p<0.01$.
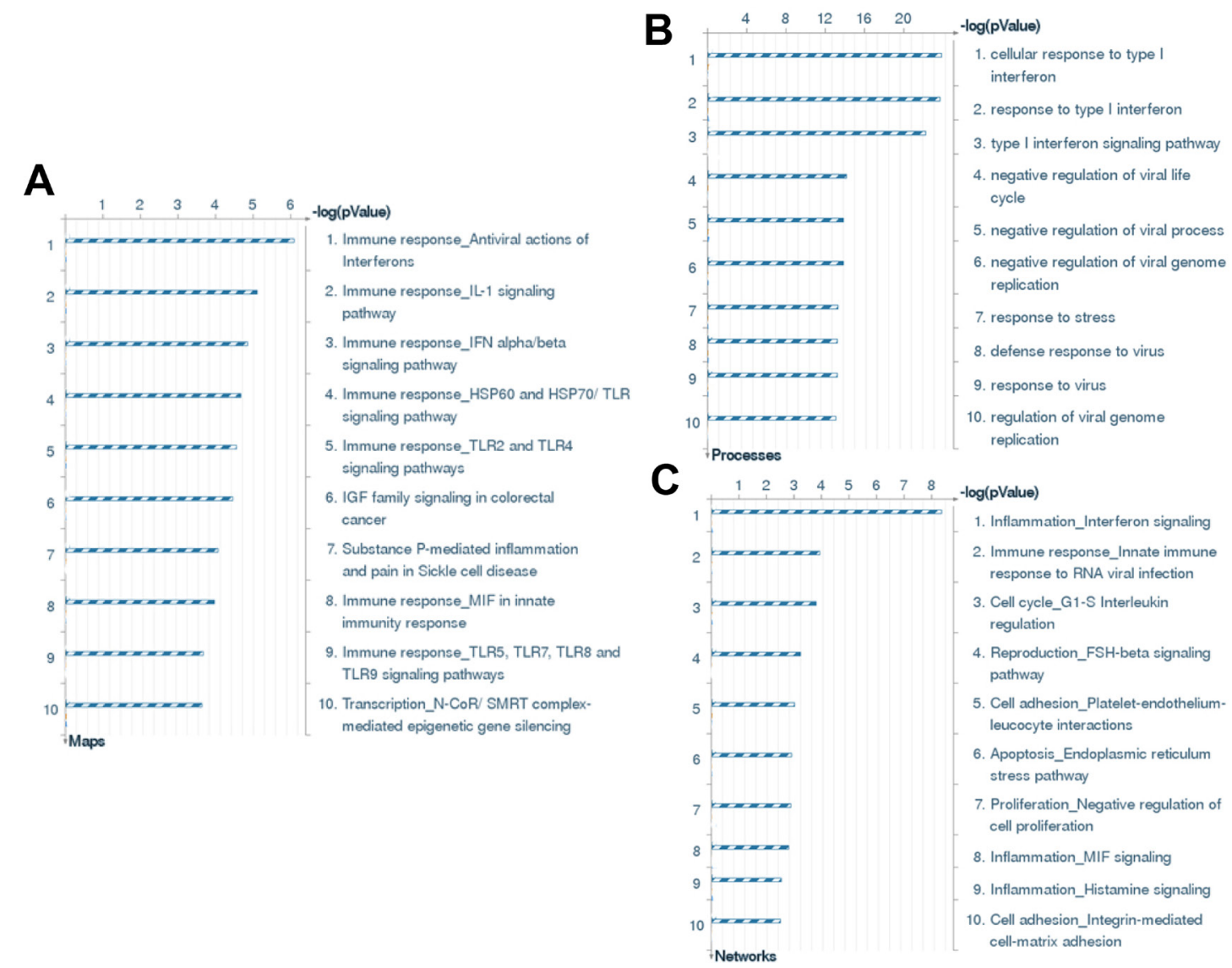

Figure 6: The enrichment analysis of gene profile alterations in KSHV-infected SiHa cells. (A-C) The enrichment analysis of gene profile significantly altered (up/down $\geq 2$ fold and $p<0.05$ ) in KSHV-infected SiHa cells was performed using the Metacore Software (Thompson Reuters) Modules: Pathway Maps (A), Gene Ontology Processes (B), and Process Networks (C). 
Table 2: The genes down-regulated within KSHV-infected SiHa cells

\begin{tabular}{|c|c|c|}
\hline Gene Symbol & Description & Ratio \\
\hline AKR1C3 & Aldo-keto reductase family 1 member $\mathrm{C} 3$ & 0.273224 \\
\hline KRT19 & Keratin, type I cytoskeletal 19 & 0.297783 \\
\hline SBSPON & Somatomedin-B and thrombospondin type- 1 domain-containing protein & 0.339295 \\
\hline SEMA3C & Semaphorin-3C & 0.350578 \\
\hline ATOH8 & Protein atonal homolog 8 & 0.370948 \\
\hline SPTSSA & Serine palmitoyltransferase small subunit A & 0.376402 \\
\hline SYNC & Syncoilin & 0.379147 \\
\hline ALDH3A1 & Aldehyde dehydrogenase, dimeric NADP-preferring & 0.383731 \\
\hline COBLL1 & Cordon-bleu protein-like 1 & 0.399284 \\
\hline DDIT4L & DNA damage-inducible transcript 4-like protein & 0.40746 \\
\hline MPZL2 & Myelin protein zero-like protein 2 & 0.408897 \\
\hline ADIRF & Adipogenesis regulatory factor & 0.416268 \\
\hline CRIP2 & Cysteine-rich protein 2 & 0.417072 \\
\hline ARL6IP5 & PRA1 family protein 3 & 0.420077 \\
\hline ALPP & Alkaline phosphatase, placental type & 0.423014 \\
\hline HEATR 5A & HEAT repeat-containing protein $5 \mathrm{~A}$ & 0.427246 \\
\hline QPCT & Glutaminyl-peptide cyclotransferase & 0.427804 \\
\hline CLIC3 & Chloride intracellular channel protein 3 & 0.431867 \\
\hline GLS & Glutaminase kidney isoform, mitochondrial & 0.445419 \\
\hline TNS3 & Tensin-3 & 0.458781 \\
\hline RHOBTB3 & Rho-related BTB domain-containing protein 3 & 0.463897 \\
\hline IDH1 & Isocitrate dehydrogenase [NADP] cytoplasmic & 0.469876 \\
\hline C14orf132 & Uncharacterized protein C14orf132 & 0.476645 \\
\hline TIMP3 & Metalloproteinase inhibitor 3 & 0.478399 \\
\hline CD14 & Monocyte differentiation antigen CD14 & 0.488419 \\
\hline CXXC5 & CXXC-type zinc finger protein 5 & 0.491275 \\
\hline SUMF1 & Sulfatase-modifying factor 1 & 0.492325 \\
\hline
\end{tabular}

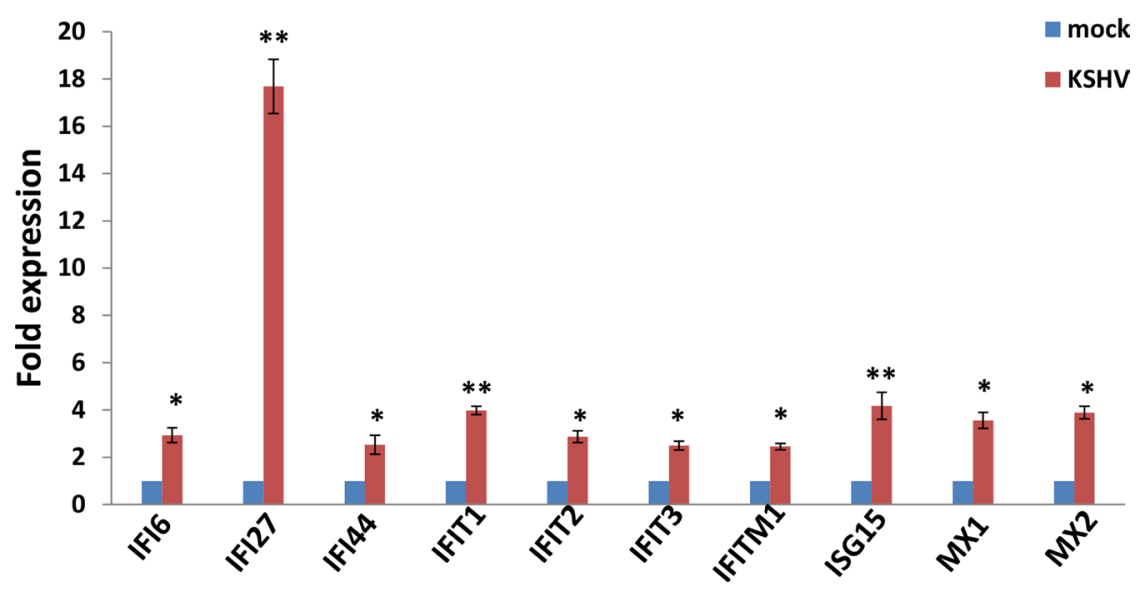

Figure 7: The up-regulation of interferon-induced genes from KSHV-infected SiHa cells. SiHa cells were infected by KSHV as described above, then gene transcripts were quantified by using qRT-PCR. The Error bars represent the S.D. for 3 independent experiments, $*=p<0.05, * *=p<0.01$. 


\section{Human cytokine/chemokine array}

The human cytokine/chemokine array was performed by using Proteome Profiler ${ }^{\mathrm{TM}}$ Array (R\&D Systems) which contains 36 different human cytokines according to the manufacturers' instructions. The density of dot-blot was scanned and quantified by using the ImageJ software.

\section{Microarray}

Microarray analysis was performed and analyzed at the Stanley S. Scott Cancer Center's Translational Genomics Core at LSUHSC. Total RNA was isolated using Qiagen RNeasy kit (Qiagen), and 500 ng of total RNA was used to synthesize dscDNA. Biotin-labeled RNA was generated using the TargetAmp-Nano Labeling Kit for Illumina Expression BeadChip (Epicentre), and hybridized to the HumanHT-12 v4 Expression BeadChip (Illumina) at $58^{\circ} \mathrm{C}$ for $16 \mathrm{~h}$. The chip was washed, stained with streptavadin-Cy3, and scanned with the Illumina BeadStation 500 and BeadScan. Using the Illumina's GenomeStudio software, we normalized the signals using the "cubic spline algorithm" that assumes that the distribution of transcript abundance is similar in all samples. The background signal was removed using the "detection $p$-value algorithm" to remove targets with signal intensities equal or lower than that of irrelevant probes (with no known targets in the human genome but thermodynamically similar to the relevant probes). The microarray experiments were performed twice for each group and the average values were used for analysis. The enrichment analysis were performed using the MetaCore Software (Thompson Reuters). The microarray original data have been submitted to Gene Expression Omnibus (GEO) database (Accession number: GSE90039).

\section{Cell proliferation and apoptosis assays}

Cell proliferation was measured by using the WST-1 assays (Roche) according to the manufacturers' instructions. Flow cytometry was used for quantitative assessment of apoptosis using the FITC-Annexin V/ propidium iodide (PI) Apoptosis Detection Kit I (BD Pharmingen).

\section{Immunoblotting}

Total cell lysates $(20 \mu \mathrm{g})$ were resolved by $10 \%$ SDS-PAGE, transferred to nitrocellulose membranes, and immunoblotted with antibodies for K8.1 (ABI) and $\beta$-Actin (Sigma) for loading controls. Immunoreactive bands were identified using an enhanced chemiluminescence reaction (Perkin-Elmer), and visualized by autoradiography.

\section{Immunofluorescence}

Cells were incubated in 1:1 methanol-acetone at $-20^{\circ} \mathrm{C}$ for fixation and permeabilization, then with a blocking reagent $(10 \%$ normal goat serum, $3 \%$ bovine serum albumin, and $1 \%$ glycine) for an additional 30 minutes. Cells were then incubated for $1 \mathrm{~h}$ at $25^{\circ} \mathrm{C}$ with 1:1000 dilution of a rat anti-LANA monoclonal antibody (ABI) followed by 1:200 dilution of a goat anti-rat secondary antibody conjugated to Texas Red (Invitrogen). For identification of nuclei, cells were subsequently counterstained with $0.5 \mathrm{mg} / \mathrm{mL} \quad$ 4',6-diamidino-2phenylindole (DAPI; Sigma) in $180 \mathrm{mM}$ Tris- $\mathrm{HCl}(\mathrm{pH}$ 7.5). Slides were washed once in $180 \mathrm{mM}$ Tris- $\mathrm{HCl}$ for 15 min and prepared for visualization using a Leica TCPS SP5 AOBS confocal microscope.

\section{Transfection assays}

For plasmid transfection, SiHa were transfected with control vector pcDNA3.1, pcDNA3.1-LANA (pcLANA) or pcDNA3.1-vFLIP (pcvFLIP) [34, 35] in 12-well plates for $48 \mathrm{~h}$ using Lipofectamine 2000 (Invitrogen) according to the manufacturer's instruction. Transfection efficiency was determined through co-transfection of a lacZ reporter construct and quantified as described previously [36].

\section{qRT-PCR}

Total RNA was isolated using the RNeasy Mini kit (QIAGEN), and cDNA was synthesized from equivalent total RNA using a SuperScript III First-Strand Synthesis SuperMix Kit (Invitrogen) according to the manufacturer's instructions. Primers used for amplification of target genes are listed in Supplementary Table 1. Amplification was carried out using an iCycler IQ Real-Time PCR Detection System, and cycle threshold $(\mathrm{Ct})$ values were tabulated in duplicate for each gene of interest in each experiment. "No template" (water) controls were used to ensure minimal background contamination. Using mean $\mathrm{Ct}$ values tabulated for each gene, and paired $\mathrm{Ct}$ values for $\beta$-actin as a loading control, fold changes for experimental groups relative to assigned controls were calculated using automated iQ5 2.0 software (Bio-rad). For amplification of human miRNAs, cDNA was synthesized using the Taqman miRNA RT kit (Applied Biosystems), and qPCR was performed using the Taqman MicroRNA Assays kit (Applied Biosystems) and a 7500 Real Time PCR System. Fold changes for microRNA were calculated using paired $\mathrm{Ct}$ values for RNU6B as recommended by the manufacturer (Applied Biosystems). The mirVana ${ }^{\mathrm{TM}}$ miRNA inhibitors for blocking miR129-5p or miR331-3p activities and the control were purchased from Invitrogen, and used according to the manufacturer's instructions. 


\section{Statistical analysis}

Significance for differences between experimental and control groups was determined using the two-tailed Student's $t$-test (Excel 8.0), and $p$ values $<0.05$ or $<0.01$ were considered significant or highly significant, respectively.

\section{ACKNOWLEDGMENTS AND FUNDING}

This work was supported by grants from a DOD Career Development Award (CA140437), the Leukemia Research Foundation, a Louisiana Clinical and Translational Science Center Pilot grant (U54GM104940 from NIH), NIH RO1s (AI091526 and AI128864) as well as awards from the National Natural Science Foundation of China (81472547, 81400164 and 81672924) and the Health Industry Project PW2013E-1 from Pudong Health Bureau of Shanghai, China. Funding sources had no role in study design, data collection and analysis, decision to publish, or preparation of the manuscript.

\section{CONFLICTS OF INTEREST}

All the authors declare no conflicts of interest.

\section{REFERENCES}

1. Munoz N, Castellsague X, de Gonzalez AB, Gissmann L. Chapter 1: HPV in the etiology of human cancer. Vaccine. 2006; 24:S3/1-10.

2. Narisawa-Saito M, Kiyono T. Basic mechanisms of highrisk human papillomavirus-induced carcinogenesis: roles of E6 and E7 proteins. Cancer Sci. 2007; 98:1505-1511.

3. Longworth MS, Laimins LA. Pathogenesis of human papillomaviruses in differentiating epithelia. Microbiol Mol Biol Rev. 2004; 68:362-372.

4. Chaitanya NC, Allam NS, Gandhi Babu DB, Waghray S, Badam RK, Lavanya R. Systematic meta-analysis on association of human papilloma virus and oral cancer. J Cancer Res Ther. 2016; 12:969-974.

5. Kim SM. Human papilloma virus in oral cancer. J Korean Assoc Oral Maxillofac Surg. 2016; 42:327-336.

6. Mallen-St Clair J, Alani M, Wang MB, Srivatsan ES. Human papillomavirus in oropharyngeal cancer: The changing face of a disease. Biochim Biophys Acta. 2016; 1866:141-150.

7. Chang Y, Cesarman E, Pessin MS, Lee F, Culpepper J, Knowles DM, Moore PS. Identification of herpesvirus-like DNA sequences in AIDS-associated Kaposi's sarcoma. Science. 1994; 266:1865-1869.

8. Cesarman E, Chang Y, Moore PS, Said JW, Knowles DM. Kaposi's sarcoma-associated herpesvirus-like DNA sequences in AIDS-related body-cavity-based lymphomas. N Engl J Med. 1995; 332:1186-1191.
9. Soulier J, Grollet L, Oksenhendler E, Cacoub P, CazalsHatem D, Babinet P, d'Agay MF, Clauvel JP, Raphael M, Degos L, et al. Kaposi's sarcoma-associated herpesviruslike DNA sequences in multicentric Castleman's disease. Blood. 1995; 86:1276-1280.

10. Diamond C, Brodie SJ, Krieger JN, Huang ML, Koelle DM, Diem K, Muthui D, Corey L. Human herpesvirus 8 in the prostate glands of men with Kaposi's sarcoma. J Virol. 1998; 72:6223-6227.

11. Howard MR, Whitby D, Bahadur G, Suggett F, Boshoff C, Tenant-Flowers M, Schulz TF, Kirk S, Matthews S, Weller IV, Tedder RS, Weiss RA. Detection of human herpesvirus 8 DNA in semen from HIV-infected individuals but not healthy semen donors. AIDS. 1997; 11:F15-19.

12. Koelle DM, Huang ML, Chandran B, Vieira J, Piepkorn M, Corey L. Frequent detection of Kaposi's sarcoma-associated herpesvirus (human herpesvirus 8) DNA in saliva of human immunodeficiency virus-infected men: clinical and immunologic correlates. J Infect Dis. 1997; 176:94-102.

13. Calabro ML, Fiore JR, Favero A, Lepera A, Saracino A, Angarano G, Schulz TF, Chieco-Bianchi L. Detection of human herpesvirus 8 in cervicovaginal secretions and seroprevalence in human immunodeficiency virus type 1-seropositive and -seronegative women. J Infect Dis. 1999; 179:1534-1537.

14. Whitby D, Smith NA, Matthews S, O'Shea S, Sabin CA, Kulasegaram R, Boshoff C, Weiss RA, de Ruiter A, Best JM. Human herpesvirus 8: seroepidemiology among women and detection in the genital tract of seropositive women. J Infect Dis. 1999; 179:234-236.

15. Pauk J, Huang ML, Brodie SJ, Wald A, Koelle DM, Schacker T, Celum C, Selke S, Corey L. Mucosal shedding of human herpesvirus 8 in men. N Engl J Med. 2000; 343:1369-1377.

16. de Sanjose S, Marshall V, Sola J, Palacio V, Almirall R, Goedert JJ, Bosch FX, Whitby D. Prevalence of Kaposi's sarcoma-associated herpesvirus infection in sex workers and women from the general population in Spain. Int $\mathbf{J}$ Cancer. 2002; 98:155-158.

17. Cotter MA, 2nd, Robertson ES. The latency-associated nuclear antigen tethers the Kaposi's sarcoma-associated herpesvirus genome to host chromosomes in body cavitybased lymphoma cells. Virology. 1999; 264:254-264.

18. Qin Z, Freitas E, Sullivan R, Mohan S, Bacelieri R, Branch D, Romano M, Kearney P, Oates J, Plaisance K, Renne R, Kaleeba J, Parsons C. Upregulation of xCT by KSHV-encoded microRNAs facilitates KSHV dissemination and persistence in an environment of oxidative stress. PLoS pathogens. 2010; 6:e1000742.

19. Zhang J, Li S, Yan Q, Chen X, Yang Y, Liu X, Wan X. Interferon-beta induced microRNA-129-5p down-regulates HPV-18 E6 and E7 viral gene expression by targeting SP1 in cervical cancer cells. PLoS One. 2013; 8:e81366.

20. Fujii T, Shimada K, Asano A, Tatsumi Y, Yamaguchi N, Yamazaki M, Konishi N. MicroRNA-331-3p Suppresses 
Cervical Cancer Cell Proliferation and E6/E7 Expression by Targeting NRP2. Int J Mol Sci. 2016; 17.

21. Li L, Xu C, Long J, Shen D, Zhou W, Zhou Q, Yang J, Jiang M. E6 and E7 gene silencing results in decreased methylation of tumor suppressor genes and induces phenotype transformation of human cervical carcinoma cell lines. Oncotarget. 2015; 6:23930-23943. doi: 10.18632/ oncotarget. 4525 .

22. Paulraj F, Abas F, Lajis NH, Othman I, Hassan SS, Naidu R. The Curcumin Analogue 1,5-Bis(2-hydroxyphenyl)-1,4pentadiene-3-one Induces Apoptosis and Downregulates E6 and E7 Oncogene Expression in HPV16 and HPV18Infected Cervical Cancer Cells. Molecules. 2015; 20:11830-11860.

23. Qin Z, DeFee M, Isaacs JS, Parsons C. Extracellular Hsp90 serves as a co-factor for MAPK activation and latent viral gene expression during de novo infection by KSHV. Virology. 2010; 403:92-102.

24. Hoppe-Seyler F, Butz K. Activation of human papillomavirus type 18 E6-E7 oncogene expression by transcription factor Sp1. Nucleic Acids Res. 1992; 20:6701-6706.

25. Demeret C, Le Moal M, Yaniv M, Thierry F. Control of HPV 18 DNA replication by cellular and viral transcription factors. Nucleic Acids Res. 1995; 23:4777-4784.

26. Qin Z, Peruzzi F, Reiss K, Dai L. Role of host microRNAs in Kaposi's sarcoma-associated herpesvirus pathogenesis. Viruses. 2014; 6:4571-4580.

27. Tsai YH, Wu MF, Wu YH, Chang SJ, Lin SF, Sharp TV, Wang HW. The M type K15 protein of Kaposi's sarcomaassociated herpesvirus regulates microRNA expression via its SH2-binding motif to induce cell migration and invasion. J Virol. 2009; 83:622-632.

28. Punj V, Matta H, Schamus S, Tamewitz A, Anyang B, Chaudhary PM. Kaposi's sarcoma-associated herpesvirusencoded viral FLICE inhibitory protein (vFLIP) K13 suppresses CXCR4 expression by upregulating miR-146a. Oncogene. 2010; 29:1835-1844.
29. Zhang Y, Wu JZ, Yang YQ, Ma R, Zhang JY, Feng JF. Expression of growthregulated oncogene1, hepatocyte growth factor, plateletderived growth factorAA and soluble Eselectin and their association with highrisk human papillomavirus infection in squamous cell carcinoma of the uterine cervix. Mol Med Rep. 2014; 10:1013-1024.

30. Dai L, Bai L, Lin Z, Qiao J, Yang L, Flemington EK, Zabaleta J, Qin Z. Transcriptomic analysis of KSHVinfected primary oral fibroblasts: The role of interferoninduced genes in the latency of oncogenic virus. Oncotarget. 2016; 7:47052-47060. doi: 10.18632/oncotarget.9720.

31. Woodworth CD. HPV innate immunity. Front Biosci. 2002; 7:d2058-2071.

32. Dai L, Trillo-Tinoco J, Cao Y, Bonstaff K, Doyle L, Del Valle L, Whitby D, Parsons C, Reiss K, Zabaleta J, Qin Z. Targeting HGF/c-MET induces cell cycle arrest, DNA damage, and apoptosis for primary effusion lymphoma. Blood. 2015; 126:2821-2831.

33. Qin Z, Dai L, Toole B, Robertson E, Parsons C. Regulation of Nm23-H1 and cell invasiveness by Kaposi's sarcomaassociated herpesvirus. J Virol. 2011; 85:3596-3606.

34. Qin Z, Dai L, Slomiany MG, Toole BP, Parsons C. Direct activation of emmprin and associated pathogenesis by an oncogenic herpesvirus. Cancer Res. 2010; 70:3884-3889.

35. Liu L, Eby MT, Rathore N, Sinha SK, Kumar A, Chaudhary PM. The human herpes virus 8-encoded viral FLICE inhibitory protein physically associates with and persistently activates the Ikappa B kinase complex. J Biol Chem. 2002; 277:13745-13751.

36. Qin Z, Kearney P, Plaisance K, Parsons CH. Pivotal advance: Kaposi's sarcoma-associated herpesvirus (KSHV)-encoded microRNA specifically induce IL-6 and IL-10 secretion by macrophages and monocytes. J Leukoc Biol. 2010; 87:25-34. 